TRANSACTIONS OF THE

AMERICAN MATHEMATICAL SOCIETY

Volume 176, February 1973

\title{
ON THE DETERMINATION OF IRREDUCIBLE MODULES BY RESTRICTION TO A SUBALGEBRA
}

BY

\section{J. LEPOWSKY(1) AND G.W. McCOLLUM}

ABSTRACT. Let $\mathcal{B}$ be an algebra over a field, $\mathcal{Q}$ a subalgebra of $\mathfrak{B}$, and $\alpha$ an equivalence class of finite dimensional irreducible $\mathbb{Q}$-modules. Under certain restrictions, bijections are established between the set of equivalence classes of irreducible $B$-modules containing a nonzero $\alpha$-primary $a$-submodule, and the sets of equivalence classes of all irreducible modules of certain canonically constructed algebras. Related results had been obtained by Harish-Chandra and R. Godement in special cases. The general methods and results appear to be useful in the representation theory of semisimple Lie groups.

1. Introduction. This paper was originally motivated by a desire to understand a certain important theorem of Harish-Chandra [4, p. 32, Theorem 2]. This theorem essentially asserts the following: Let $g$ be a real semisimple Lie algebra and $\notin \subset g$ the fixed subalgebra of a Cartan involution of $g$. Let $V$ be an irreducible

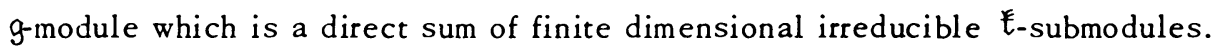
Then $V$ is determined up to equivalence by the knowledge of the action of $\mathfrak{E}$ and of the centralizer of $E$ in the universal enveloping algebra of $g$ on any one of the nonzero primary $E$-submodules of $V$. In this paper, we shall generalize, simplify and sharpen Harish-Chandra's argument.

In proving his theorem, Harish-Chandra uses infinitesimal characters, and relies on the finiteness of a certain module, which he proves in [3, p. 195, Theorem 1]. Thus he uses some very special properties of the pair $(g, \mathfrak{E})$. (This does, however, enable him to obtain a certain finiteness corollary [4, p. 36, Corollary 1] which we do not attempt to generalize.) We shall replace the pair $(g, \mathfrak{E})$ by the pair $(\mathfrak{b}, a)$, where $\mathfrak{b}$ is an arbitrary (possibly infinite dimensional) Lie algebra over a field of characteristic zero, and $a$ is any Lie subalgebra of $b$ which has an $a$-invariant complement in $\mathfrak{b}$ on which the natural representation of $a$ is a

Received by the editors December 18, 1971.

AMS (MOS) subject classifications (1970). Primary 16A64, 17B35, 17B65, 20G05, $22 \mathrm{E} 45$.

Key words and phrases. Irreducible module, irreducible representation, finitely semisimple module, absolutely irreducible module, primary submodule, extension of submodules, Lie algebra, universal enveloping algebra, Poincaré-Birkhoff-Witt theorem, simple ring, full matrix algebra.

(1) Partially supported by NSF GP 28323. 
direct sum of finite dimensional irreducible submodules. In $\$ 2$, we develop the relevant properties of such a pair. These properties are essentially already known (cf. [3, pp. 195-196] for a special case).

In $\$ 3$, we consider quite generally an arbitrary algebra $\mathcal{B}$ and subalgebra $\mathfrak{A}$ over an arbitrary field $(\mathscr{B}$ and $\mathscr{A}$ will be specialized to the universal enveloping algebras of $\mathfrak{b}$ and $a$, respectively). In this setting, we define and develop the general properties of the key subspaces $A^{\beta, \alpha}$ of $B$. Here $\beta$ and $\alpha$ are equivalence classes of finite dimensional irreducible $\mathfrak{Q}_{\text {-modules, }}$ and $A^{\beta, \alpha}$ is the subspace of $\mathscr{B}$ which sends the $\alpha$-primary subspace of any $\mathscr{B}$-module (regarded as an Q-module by restriction) into the $\beta$-primary subspace. The definition of the se spaces in the "diagonal" cases $A^{a, a}$ was suggested by Harish-Chandra's spaces $A[4$, p. 33] and $A(\Im)[4$, p. 50].

Let $\alpha$ be an equivalence class of finite dimensional irreducible $\mathbb{Q}$-modules, and let $\mathscr{g}^{\alpha}$ be the annihilator in $\mathcal{Q}$ of any module in $\alpha$. In $\$ 4$, by adding the assumption that $\mathfrak{B} / \mathcal{B}^{\alpha}$, regarded as an $\mathfrak{Q}$-module, is a direct sum of finite dimensional irreducible submodules, we establish a bijection betwe en the set of equivalence classes of all irreducible $\mathfrak{B}$-modules containing a nonzero $\alpha$-primary $\mathfrak{A}$-submodule and the set of equivalence classes of all irreducible modules of the algebra $A^{a, a} / B^{a}$ (Theorem 4.9). This is a generalization of part of Harish-Chandra's argument (cf. [4, p. 34]). One feature of our argument is that in place of HarishChandra's use of Zorn's lemma [4, p. 34], we explicitly construct the appropriate maximal left ideal of $\mathcal{B}$, in a general module-theoretic setting (Propositions 4.6 and 4.7).

We begin $\$ 5$ with three known general lemmas concerning full matrix algebras. We then make the assumptions of $\$ 2$, together with two additional ones. This enables us to show that the algebra $A^{\alpha, \alpha / \beta g^{\alpha}}$ mentioned above is the tensor product of a full matrix algebra with the algebra $\mathcal{B}^{a} / \mathcal{B}^{a} \cap \mathcal{B}^{a}$, where $\mathcal{B}^{a}$ is the centralizer of $a$ in the universal enveloping algebra $\mathcal{B}$ of $\mathfrak{b}$ (Theorem 5.4). We can then prove the main result (Theorem 5.5), which establishes a bijection between the set of equivalence classes of all irreducible $B$-modules containing a nonzero $a$-primary $\mathfrak{Q}$-submodule and the set of equivalence classes of all irreducible modules of $\mathcal{B}^{a} / \mathcal{B}^{a} \cap B^{a}$ (Harish-Chandra proves only the injectivity in his special case; see [4, p. 32, Theorem 2] and [4, p. 36, Corollary 2]).

We remark that Theorem 5.5 may be regarded as an algebraic analogue of certain results of $\mathrm{R}$. Godement [2, Theorems 8, 9 and 12] on generalized spherical functions.

We shall consider applications of our results in later papers (see [7]).

2. The Lie algebra setting. Let $F$ be a field of characteristic zero, $b$ a Lie algebra over $F, a \subset b$ a Lie subalgebra of $\mathfrak{b}$, and $c \subset b$ an $a$-invariant 
complement for $a$ in $b$ such that the natural representation of $a$ on $c$ is finitely semisimple (that is, it is semisimple and all the irreducible components are finite dimensional).

Let $\mathfrak{B}$ (resp., $\mathfrak{A}$ ) denote the universal enveloping algebra of $\mathfrak{b}$ (resp., $a$ ), so that we may regard $\mathscr{A} \subset \mathcal{B}$ in the natural way. Then $\mathfrak{b}$-modules (resp., a-modules) are identified with $\mathfrak{B}$-modules (resp., $\mathfrak{Q}$-modules).

Lemma 2.1. The tens or product (over F) of two finitely semisimple represen. tations of $a$ is again finitely semisimple.

Proof. It is sufficient to show that if $\pi$ and $\rho$ are finite dimensional irreducible representations of $a$, then $\pi \otimes \rho$ is a semisimple representation of $a$. Let $i_{\pi}$ (resp., $i_{\rho}$ ) denote the kernel of $\pi$ (resp., $\rho$ ) in $a$. Now $a_{1}=a / i_{\pi} \cap i_{\rho}$ is a finite dimensional Lie algebra, and $\pi$ and $\rho$ can be regarded as irreducible representations of $a_{1}$. Then $\pi \otimes \rho$ is a semisimple representation of $a_{1}$, since $F$ has characteristic zero (see $[1$, p. 83 , Corollaire 1]). Thus $\pi \otimes \rho$ is a semisimple representation of a. Q.E.D.

For every vector space $V$ (over $F$ ), let $S(V)$ denote the symmetric algebra over $V$. Let $\lambda: S(\mathfrak{b}) \rightarrow \mathbb{B}$ denote the "symmetrization" mapping, that is, the unique linear isomorphism such that

$$
\lambda\left(x_{1} \ldots x_{n}\right)=\frac{1}{n !} \sum_{\sigma} x_{\sigma(1)} \cdots x_{\sigma(n)}
$$

for all nonnegative integers $n$ and all $x_{1}, \ldots, x_{n} \in \mathbb{B}$ (see $[1, \S 2.7]$ ). Here $\sigma$ ranges over all permutations of $\{1, \cdots, n\}$, the product on the left is taken in $S(\mathcal{B})$, and the products on the right are taken in $\mathcal{B}$. It is interesting to note that $\lambda$ may also be defined as the unique linear map from $S(\mathfrak{G})$ to $\mathbb{B}$ such that $\lambda\left(x^{n}\right)=$ $x^{n}$ for all $x \in \mathfrak{b}, n \geq 0$, since the powers $x^{n}(x \in \mathfrak{b}, n \geq 0) \operatorname{span} S(\mathfrak{b})$. We note that $\lambda$ is defined on $S(a)$ and $S(c)$ by regarding $S(a) \subset S(b)$ and $S(c) \subset S(b)$.

Now $S(\mathfrak{b})$ and $B$ are both $\mathfrak{b}$-modules, by unique extension by derivations of the adjoint representation of $\mathfrak{b}$ on itself. Moreover, $\lambda$ is a $\mathfrak{b}$-module map (see [1, \$2.8]).

The natural action of $a$ on $c$ extends uniquely to an action $\rho$ of $a$ as derivations of $S(c)$. We assert that $\rho$ is finitely semisimple. Indeed, the action of $a$ on $c$ is finitely semisimple by hypothesis, and so the natural action of $a$ as derivations of the tensor algebra over $c$ is finitely semisimple, by Lemma 2.1. But since $\rho$ is a quotient of this representation, $\rho$ is finitely semisimple.

Let $\mathcal{I}$ be a left ideal of $\mathfrak{Q}$, and let $\sigma$ denote the natural representation of $a$ on $\mathfrak{Q} / \mathcal{G}$ induced by left multiplication. Let $\tau$ denote the natural representation of $a$ on $B / B g$ induced by left multiplication. We shall relate $\rho, \sigma$ and $\tau$ in Lemmas 2.3 and 2.4 . 
Lemma 2.2 (cf. [3, p. 193, Lemma 12]). The map $f: S(c) \otimes \mathbb{A} \rightarrow \mathfrak{B}$ given by $x \otimes y \mapsto \lambda(x) y$ is a linear is omorphism.

Proof. For every vector space $V$ (over $F$ ), let $T(V)$ denote the tensor algebra over $V$, and for every $n \geq 0$, let $T^{n}(V)$ denote the $n$th graded subspace of $T(V)$. Let $\Sigma^{n}(V)$ denote the space of symmetric tensors in $T^{n}(V)$, that is, the tensors left fixed by the natural action of the symmetric group on $n$ letters on $T^{n}(V)$, and let

$$
\Sigma(V)=\coprod_{n=0}^{\infty} \Sigma^{n}(V) \subset T(V) .
$$

Also, let $S^{n}(V)$ denote the $n$th graded subspace of $S(V)$.

We regard $T(a) \subset T(\mathfrak{b}), T(c) \subset T(\mathfrak{b}), S(a) \subset S(b)$ and $S(c) \subset S(\mathfrak{b})$. The map $g: S(c) \otimes S(a) \rightarrow S(\mathfrak{b})$ given by $x \otimes y \mapsto x y$ is an algebra isomorphism. Let

$$
\pi: T(\mathfrak{b}) \rightarrow S(\mathfrak{b})
$$

be the natural projection homomorphism. Then the restrictions $(\pi \mid T(c)): T(c) \rightarrow S(c)$ and $(\pi \mid T(a)): T(a) \rightarrow S(a)$ are the natural projection homomorphisms for $c$ and $a$, respectively, so that the restrictions

$$
\pi_{1}: \Sigma(c) \rightarrow S(c), \quad \pi_{2}: \Sigma(a) \rightarrow S(a)
$$

of $\pi$ are linear isomorphisms.

Let

$$
W=\Sigma(c) \otimes \Sigma(a) \subset T(b),
$$

and, for each $n \geq 0$, let $W^{n}=W \cap T^{n}(\mathfrak{b})$, so that

$$
W=\coprod_{n=0}^{\infty} W^{n} .
$$

Then $(\pi \mid W): W \rightarrow S(\mathfrak{B})$ can be factored as

$$
\pi \mid W=g \circ\left(\pi_{1} \otimes \pi_{2}\right) .
$$

Hence $\pi \mid W$ is a linear isomorphism, and so

$$
\pi \mid W^{n}: W^{n} \rightarrow S^{n}(\mathfrak{b})
$$

is a linear isomorphism for each $n \geq 0$. Thus it follows from [1, p. 33, Corollaire 1] (essentially the Poincaré-Birkhoff-Witt theorem) that $\phi: W \rightarrow \mathscr{B}$ is a linear isomorphism, where $\phi$ is the restriction to $W$ of the natural projection map from $T(\mathfrak{b})$ onto $\mathfrak{B}$. But $(\phi \mid \Sigma(a)): \Sigma(a) \rightarrow \mathbb{A}$ is a linear isomorphism, again by $[1, \mathrm{p}$. 33, Corollaire 1 ], so that

$$
\phi \circ\left(\left(\pi_{1}\right)^{-1} \otimes(\phi \mid \Sigma(a))^{-1}\right): S(c) \otimes \mathbb{Q} \rightarrow \mathfrak{B}
$$


is a linear isomorphism. Since $\lambda \mid S(c)=\phi \circ\left(\pi_{1}\right)^{-1}$ and $\phi$ is a homomorphism, we have the lemma. Q.E.D.

Lemma 2.3. The map $1: S(c) \otimes \mathfrak{A} / \mathscr{I} \rightarrow \mathfrak{B} / \mathfrak{B g}$ given by $x \otimes(y+\mathscr{I}) \mapsto \lambda(x) y+$ $\mathfrak{B g}(x \in S(c), y \in \mathbb{Q})$ is a linear isomorphism.

Proof. From the exact sequence

we have that the sequence

$$
0 \rightarrow \mathfrak{I} \rightarrow \mathbb{A} \rightarrow \mathbb{A} / \mathscr{I} \rightarrow 0
$$

$$
0 \rightarrow S(c) \otimes I \rightarrow S(c) \otimes \mathbb{Q} \rightarrow S(c) \otimes \mathbb{A} / \mathscr{I} \rightarrow 0
$$

is exact. The lemma follows from the isomorphisms

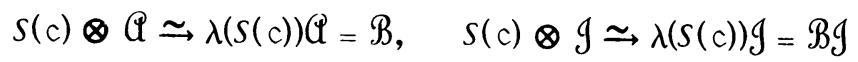

given by Lemma 2.2. Q.E.D.

Lemma 2.4 (cf. [3, p. 195, The orem 1, first assertion]). The isomorphism । of Lemma 2.3 is an a-module map with respect to $\rho \otimes \sigma$ and $\tau$. In particular, if $\sigma$ is finitely semisimple, then $\tau$ is finitely semisimple.

Proof. Let $a \in a, x \in S(c)$ and $y \in \mathbb{Q}$. Then

$$
\begin{aligned}
& \iota(a \cdot(x \otimes(y+\mathcal{G})))=\iota(a \cdot x \otimes(y+\mathcal{I})+x \otimes(a y+\mathscr{g})) \\
& =\lambda(a \cdot x) y+\lambda(x) a y+\mathfrak{B S}=(a \cdot \lambda(x)) y+\lambda(x) a y+\mathfrak{B g} \\
& =a \lambda(x) y-\lambda(x) a y+\lambda(x) a y+\mathfrak{B g} \\
& =a \lambda(x) y+\mathfrak{B} \mathcal{S}=a \cdot \iota(x \otimes(y+\mathcal{G})),
\end{aligned}
$$

proving the first statement. The second statement follows from Lemma 2.1. Q.E.D.

3. The spaces $A^{\beta, a}$. We now generalize the setting of $\$ 2$. Let $F$ be any field. Let $B$ be an (associative) algebra (with 1 ) over $F$, and $\mathscr{A} \subset \mathcal{B}$ a subalgebra of $\mathfrak{B}$. Let $\hat{\mathfrak{Q}}$ denote the set of equivalence classes of finite dimensional simple $\mathbb{Q}$-modules (or, equivalently, finite dimensional irreducible representations of $\mathfrak{Q}$ ). For any $\alpha \in \hat{\mathbb{A}}$ and any $\mathfrak{Q}$-module $V$, we denote by $V_{a}$ the $\alpha$-primary subspace of $V$, that is, the sum of all the simple submodules of $V$ in the class $\alpha$. We shall regard $\mathfrak{B}$-modules as $\mathfrak{Q}$-modules by restriction. For every $\alpha \in \hat{\mathbb{C}}$, we denote by $g^{a}$ the kernel in $\mathcal{P}$ of any representation in the class $\alpha$, so that $g^{a}$ is a two-sided ideal of $\mathbb{Q}$.

Lemma 3.1. Let $a \in \hat{\mathbb{A}}$, and let $V$ be an $\mathfrak{Q}$-module. Then $V_{a}$ is precisely the annibilator of $g^{a}$ in $V$.

Proof. Let $V_{1}$ be the annihilator of $g^{a}$ in $V$. Clearly, $V_{\alpha} \subset V_{1}$. Now $\mathbb{G} / \mathscr{G}^{a}$ is a simple ring. Indeed, let $W$ be an $\mathbb{Q}$-module in the class 
$\alpha$, so that $W$ is a simple faithful module for the ring $\mathbb{Q} / \mathscr{g}^{a}$. Let $K=$ End $_{Q W}$, so that $K$ is a division ring, and $W$ is finite dimensional over $K$. By Wedderburn's the orem, $\mathfrak{Q} / g^{a} \simeq$ End $_{K} W$, which is a simple ring.

Now the unique equivalence class of simple $\mathfrak{Q} / \mathscr{g}^{a}$-modules is the class induced by $a$. Since $V_{1}$ is an $\mathfrak{Q} / \mathscr{G}^{\alpha}$-module, $V_{1}$ is an $\alpha$-primary $\mathscr{Q}$-module. Hence $V_{1} \subset$ $V_{a}$. Q.E.D.

Let $a \in \hat{A}$. The left ideal $\mathfrak{B g}^{\alpha}$ of $\mathfrak{B}$ can be characterized as follows:

Proposition 3.2. The le $/ t$ ideal $\mathfrak{B g}^{\alpha}$ of $\mathfrak{B}$ is precisely the subset of $\mathfrak{B}$ which annibilates $V_{a}$ for every B.module $V$, or, alternatively, which annibilates $V_{a}$ in the special case in which $V=\mathscr{B} / \mathfrak{B g}^{a}$, regarded as the $\mathfrak{B}$-module induced by left multiplication.

Proof. To show that the annihilator of $\left(\mathscr{B} / \mathcal{B g}^{\alpha}\right)_{\alpha}$ in $B$ is contained in $\mathcal{B g}^{\alpha}$, we have

$$
1+B \mathfrak{g}^{a} \in\left(\mathscr{B} / \mathcal{B g}^{a}\right)_{a}
$$

by Lemma 3.1. Hence if $x \in \mathscr{B}$ annihilates $\left(\mathscr{B} / \mathscr{B g}^{\alpha}\right)_{a}$, then $x \in \mathfrak{B g}^{\alpha}$. Q.E.D.

Fix $\alpha, \beta \in \hat{\mathbb{U}}$. We define

$$
A^{\beta, \alpha}=\left\{x \in \mathscr{B} \mid g^{\beta} x \subset \mathfrak{B g}^{\alpha}\right\}=\left\{x \in \mathscr{B} \mid \mathfrak{B g}^{\beta} x \subset \mathfrak{B g}^{\alpha}\right\} .
$$

We can characterize $A^{\beta, \alpha}$ in two ways, given by the next two propositions:

Proposition 3.3. $A^{\beta, \alpha}$ is precisely the subset of $B$ which transforms $V_{a}$ into $V_{\beta}$ for every $\mathfrak{B}$-module $V$, or, alternatively, which transforms $V_{a}$ into $V_{\beta}$ in the special case in which $V=\mathcal{B} / \mathfrak{B g}^{a}$.

Proof. $A^{\beta, a}$ transforms $V_{a}$ into $V_{\beta}$ by Lemma 3.1. Conversely, if $x \in \mathfrak{B}$ transforms $\left(\mathscr{B} / \mathcal{B g}^{\alpha}\right)_{a}$, into $\left(\mathscr{B} / \mathcal{B g}^{\alpha}\right)_{\beta}$, then $x \cdot\left(1+\mathcal{B g}^{\alpha}\right)=x+\mathfrak{B g}^{\alpha} \in\left(\mathscr{B} / \mathcal{B g}^{\alpha}\right)_{\beta}$, so that $\mathscr{I}^{\beta} x \subset B^{a}$. Q.E.D.

Proposition 3.4. $\mathcal{B g}^{\alpha} \subset A^{\beta, \alpha}$, and $A^{\beta, \alpha} / \mathcal{B g}^{\alpha}=\left(\mathscr{B} / \mathcal{B g}^{\alpha}\right)_{\beta}$ (regarding $\mathfrak{B} / \mathcal{B} g^{\alpha}$ as a B-module).

Proof. Immediate from Lemma 3.1. Q.E.D.

Proposition 3.5. We bave $A^{\gamma, \beta} A^{\beta, a} \subset A^{\gamma, a}$ for all $\alpha, \beta, \gamma \in \hat{\mathbb{G}}$.

Proof. Clear from either the definition or from Proposition 3.3. Q.E.D.

The following result concerning the special case $A^{\alpha, \alpha}(\alpha \in \hat{\mathbb{A}})$ is clear:

Proposition 3.6. $A^{a, a}$ is a subalgebra of $\mathcal{B}$, and is the normalizer in $\mathfrak{B}$ of the left ideal $\mathfrak{B g}^{a}$ of $\mathfrak{B}$, that is, $A^{a, a}$ is the largest subalgebra of $\mathfrak{B}$ wbicb con. tains $B^{a}$ as a two-sided ideal. Moreover, $A^{a, a} / B^{a}$ is an algebra which acts naturally on $V_{a}$ for every $ß$-module $V$. 
Remark 3.7. The definition of $A^{\alpha, \alpha}$ was suggested by [4, pp. 33, 50], as indicated in the Introduction.

We give another interpretation of the $\mathscr{B}$-module $\mathscr{B} / \mathcal{B g}^{a}$ :

Proposition 3.8. The map $f: \mathcal{B} \otimes_{\mathbb{Q}} \mathfrak{A} / \mathscr{g}^{a} \rightarrow \mathfrak{B} / \mathcal{B g}^{a}$ given by $x \otimes\left(y+g^{a}\right) \mapsto$ $x y+\mathfrak{B g}^{\alpha}(x \in \mathfrak{B}, y \in \mathfrak{Q})$ is a $\mathfrak{B}$-module isomorphism. (Here $\mathfrak{B}$ is regarded as a left B-module and a right $\mathfrak{Q}$-module.)

Proof. The map $f$ is clearly well defined and is a $\mathfrak{B}$-module map. Conversely, the map $g: \mathfrak{B} / \mathfrak{B g}^{\alpha} \rightarrow \mathfrak{B} \otimes_{\mathbb{Q}} \mathfrak{A} / \mathscr{g}^{\alpha}$ given by $x+B^{a} \mapsto x \otimes 1(x \in \mathfrak{B})$ is well defined, is a $\mathfrak{B}$-module map, and is a left and right inverse of $f$. Q.E.D.

4. Extension of submodules and ideals; application to irreducible modules. We retain the notation and assumptions of $\$ 3$. In addition, we assume that the natural representation of $\mathcal{A}$ on $\mathcal{B} / \mathcal{B g}^{\alpha}$ is finitely semisimple for a certain $\alpha \in \hat{\mathbb{U}}$ fixed throughout this section. This holds in particular (for arbitrary $\alpha$ ) under the assumptions of $\$ 2$, by Lemma 2.4 .

Remark 4.1. In view of Proposition 3.4, the new assumption implies that

$$
\mathscr{B} / \mathcal{B g}^{\alpha}=\coprod_{\beta \in \hat{\mathrm{Q}}} A^{\beta, a} / \mathfrak{B g}^{\alpha}
$$

and hence that $B=\Sigma_{\beta \in \hat{\mathbb{Q}}} A^{\beta, \alpha}$ and that

for all $\beta \epsilon \hat{\mathbb{Q}}$.

$$
A^{\beta, a} \cap \sum_{\gamma \in \hat{\mathbb{Q}} ; \gamma \neq \beta} A^{\gamma, \alpha}=\left(\mathfrak{B}^{a}\right.
$$

Proposition 4.2. Let $V$ be an irreducible $B$-module sucb that $V_{\alpha} \neq 0$, or more generally, let $V$ be a $\Re$-module generated by $V_{\alpha}$. Then $V$ is finitely semisimple under $\mathbb{Q}$.

Proof. Remark 4.1 and Proposition 3.3 imply that

$$
B \cdot V_{\alpha} \subset \coprod_{B \in \hat{\mathbb{Q}}} V_{\beta} \cdot \quad \text { Q.E.D. }
$$

Remark 4.3. One can easily generalize Proposition 4.2 as follows: Let

$$
\Gamma=\left\{\gamma \in \hat{i} \mid: B /: B g^{\gamma} \text { is finitely semisimple under }(\mathcal{P}\}\right. \text {. }
$$

Let $V$ be a $\mathbb{B}$-module. Then

$$
\text { B. } \coprod_{\gamma \in \Gamma} V_{\gamma} \subset \coprod_{\beta \in \hat{\mathbb{Q}}} V_{\beta} .
$$

In particular, if $\Gamma=\hat{\mathrm{\varphi}}$ (for example, under the assumptions of $\S 2$ ), then $\mathrm{II}_{\beta \in \hat{\mathrm{Q}}} V_{\beta}$ is a $B$-submodule of $V$. 
Proposition 4.4. Let $V$ be a $\Re$-module, let $\beta \in \hat{\mathbb{Q}}$ and let $S$ be a subset of $V_{a}$. Then $(\mathcal{B} \cdot S) \cap V_{\beta}=A^{\beta, \alpha} \cdot S$.

Proof. Immediate from Remark 4.1 and Proposition 3.3. Q.E.D.

Proposition 4.5 (cf. [4, p. 33]). Let $V$ be an irreducible B-module and suppose that $V_{a} \neq 0$. Then $V_{a}$ is invariant and irreducible under $A^{a, a}$. More generally, if $V$ is not necessarily irreducible, then $V_{\alpha}$ is $A^{a, \alpha}$-irreducible if and only if $V_{a} \neq \delta$ and every $B$-submodule of $V$ which meets $V_{a}$ contains $V_{a}$.

Proof. Suppose that $V$ is not necessarily irreducible, that $V_{\alpha} \neq 0$ and that every $\mathcal{B}$-submodule of $V$ which meets $V_{\alpha}$ contains $V_{\alpha}$. Let $W$ be a nonzero $A^{\alpha, a}$ submodule of $V_{a}$. Then $(\Re \cdot W) \cap V_{a}=A^{a, \alpha} \cdot W=W$ by Proposition 4.4, and so $\mathfrak{B} \cdot W \supset V_{a}$ by hypothesis. Thus $W=V_{a}$, so that $V_{a}$ is $A^{a, a}$-irreducible. The rest of the proposition is clear. Q.E.D.

Let $V$ be a $\Re$-module and let $W$ be an $A^{\alpha, a}$-submodule of $V_{\alpha}$. Then we define an extension of $W$ to be a $B$-submodule of $V$ whose intersection with $V_{\alpha}$ is $W$. The following is a "going-up" theorem:

Proposition 4.6. Let $V$ be a $B$-module, and let $W$ be an $A^{a, a}$-submodule of $V_{\alpha}$. Then $W$ bas a smallest extension $W^{\min }$, and $W^{\min }=\mathfrak{B} \cdot W$. Moreover, $W^{\text {min }}$ is contained in every extension of every $A^{a, a}$-submodule of $V_{a}$ containing $W$. Now let $V$ be finitely semisimple under $\mathbb{Q}$, and let $P: V \rightarrow V_{a}$ be the natural projection map with respect to the semisimple decomposition. Then $W$ bas a largest extension $W^{\max }$, and

$$
\begin{aligned}
W^{\max } & =\left\{v \in V \mid(\Re \cdot v) \cap V_{a} \subset W\right\} \\
& =\{v \in V \mid P(\Re \cdot v) \subset W\} .
\end{aligned}
$$

Moreover, $W^{\max }$ contains every extension of every $A^{a, a}$-submodule of $W$.

Proof. The assertions about $W^{\text {min }}$ are clear by taking $\beta=\alpha$ and $S=W$ in Proposition 4.4. Let $V$ be finitely semisimple under $\mathfrak{Q}$. Now the right-hand side of $(*)$ equals the right-hand side of $(* *)$, since $\mathcal{B} \cdot v$ is an $\mathbb{Q}$-submodule of $V$, and so $(\Re \cdot v) \cap V_{a}=P(\Re \cdot v)$ for all $v \in V$. Let $X$ be the right-hand side of (*). Then $X$ is clearly a $\mathfrak{B}$-submodule of $V$, by $(* *)$. If $w \in W$, then

$$
(\mathscr{B} \cdot w) \cap V_{a}=A^{a, a} \cdot w \subset W
$$

by Proposition 4.4, so that $X$ is an extension of $W$. If $Y$ is an extension of an $A^{a, a}$-submodule of $W$, then for all $y \in Y$,

$$
(\mathscr{B} \cdot y) \cap V_{a} \subset Y \cap V_{a} \subset W,
$$

so that $y \in X$. Thus $W^{\max }$ is the largest extension of $W$, and the last assertion holds. Q.E.D. 
We now apply Proposition 4.6 to the case $V=\mathscr{B} / \mathcal{B g}^{\alpha}$. Let $M(\alpha)$ be the set of maximal left ideals of $B$ containing $B^{\alpha}$, and let $L(\alpha)$ be the set of maximal left ideals of $A^{a, a}$ containing $B^{\alpha}$. For every $\mathbb{M} \in M(\alpha)$, let $\phi(M)=\mathbb{M} \cap A^{\alpha, a}$. Let $\pi: \mathcal{B} \rightarrow \mathcal{B} / \mathcal{B g}^{\alpha}$ be the quotient map, and let $P: B / B g^{\alpha} \rightarrow A^{\alpha, \alpha} / \mathcal{B g}^{\alpha}$ be the pro-

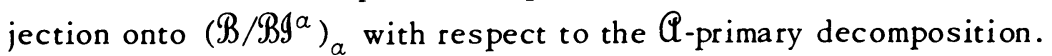

Proposition 4.7 (cf. [4, pp. 33-34]). We bave $\phi(M(\alpha)) \subset L(\alpha)$, and $\phi: M(\alpha) \rightarrow$ $L(\alpha)$ is a bijection. If $\mathcal{Q} \in L(\dot{\alpha})$, then

$$
\begin{aligned}
& \phi^{-1}(\mathcal{Q})=\left\{x \in \mathscr{B} \mid\left(\Re_{x}\right) \cap A^{\alpha, a} \subset \mathcal{L}^{\infty}\right\} \\
& =\left\{x \in \mathcal{B} \mid(\mathfrak{B} \cdot \pi(x)) \cap \pi\left(A^{a, a}\right) \subset \pi(\mathscr{Q})\right\} \\
& =\{x \in \mathfrak{B} \mid P(\mathscr{B} \cdot \pi(x)) \subset \pi(\mathcal{Q})\}=\pi^{-1}\left(\pi\left(\mathcal{Q}^{\max }\right)\right.
\end{aligned}
$$

(see Proposition 4.6). Moreover, $\phi^{-1}(\mathcal{Q})$ contains every left ideal $\mathbb{M}$ of $\mathbb{B}$ such that $\mathbb{R} \cap A^{a, a} \subset \mathcal{Q}$.

Proof. Let $\mathbb{M} \in M(\alpha)$, so that $\mathbb{B} / \mathbb{M}$ is an irreducible $\mathbb{B}$-module. Since $1+\mathbb{M} \epsilon$ $(\Re / M)_{\alpha},(\Re / M)_{\alpha} \neq 0$, and the annihilat or of $1+\mathbb{M}$ in $A^{\alpha, a}$ is $\mathbb{M} \cap A^{\alpha, a}$. But by Proposition 4.5, $(\Re / M)_{\alpha}$ is $A^{\alpha, \alpha}$-irreducible. Thus $\mathbb{M} \cap A^{\alpha, \alpha} \in L(\alpha)$, so that $\phi(M(\alpha)) \subset$ $L(\alpha)$.

Now let $\mathcal{L} \in L(\alpha)$, so that $\pi(\mathscr{Q})$ is a maximal proper $A^{a, a}$-submodule of $\pi\left(A^{a, a}\right)$. Then $\pi(\mathcal{Q})^{\max }$ (see Proposition 4.6) is a maximal proper $\mathfrak{B}$-submodule of $\pi(\mathfrak{B})$. Indeed, $\pi(£)^{\max }$ is the largest extension of $\pi(£)$, and so any strictly larger $\Re$-submodule of $\pi(\mathscr{B})$ would contain $\pi(1)$, and hence would equal $\pi(\Re)$. Thus $\pi^{-1}\left(\pi(\mathscr{Q})^{\max }\right) \epsilon$ $M(\alpha)$, and $\pi^{-1}\left(\pi(£)^{\max }\right) \cap A^{\alpha, \alpha}=\mathcal{L}$. Conversely, if $M \in M(\alpha)$ and $M \cap A^{\alpha, \alpha}=\mathscr{L}$, then $\pi(M)$ is clearly a maximal extension of $\pi(\varrho)$, so that $\pi(\pi)=\pi(£)^{\max }$. Thus $\phi$ is a bijection, and

$$
\phi^{-1}\left(\mathcal{\complement}^{\mathcal{D}}\right)=\pi^{-1}\left(\pi\left(\Re^{\mathrm{Pax}}\right) .\right.
$$

The remaining formulas for $\phi^{-1}(£)$ follow from Proposition $4.6(*),(* *)$, and the last statement follows from the last statement of Proposition 4.6. Q.E.D.

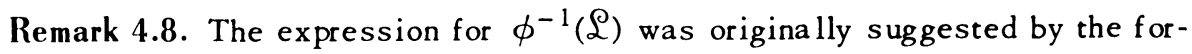
mula for $L_{\lambda}$ in $[6$, p. 632] and the formula for $m$ in $[2$, p. 513, Lemma 8].

Theorem 4.9 (cf. [4, p. 34]). The correspondence $V \mapsto V_{\alpha}$, where $V$ ranges through the irreducible $\mathfrak{B}$-modules such that $V_{a} \neq 0$, induces a bijection from the set of equivalence classes of all such $\mathfrak{B}$-modules with the set of equivalence classes of all irreducible $\Lambda^{\alpha, \alpha} / B^{\alpha}$-modules.

Proof. If $V$ is an irreducible $\mathfrak{B}$-module such that $V_{a} \neq 0$, then $V_{a}$ is an irreducible $A^{\alpha, a}$-module by Proposition 4.5, and hence naturally induces an irreducible $A^{\alpha, a} / B^{\alpha} g^{\alpha}$-module. The equivalence class of $V$ clearly determines that of $V_{\alpha}$.

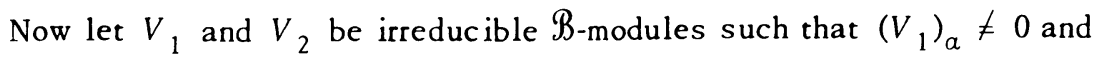
$\left(V_{2}\right)_{\alpha} \neq 0$, and suppose $f:\left(V_{1}\right)_{\alpha} \rightarrow\left(V_{2}\right)_{\alpha}$ is an $A^{\alpha, a}$-isomorphism. Choose a 
nonzero element $v \in\left(V_{1}\right)_{a}$. Let $\mathbb{M}_{1}$ (resp., $\mathbb{M}_{2}$ ) be the annihilator of $v$ (resp., $f(v))$ in $\mathfrak{B}$, so that $\mathbb{M}_{i} \in M(a)(i=1,2)$. Let $\mathcal{E}$ be the annihilator of $v$ in $A^{a, a}$ Then $\mathfrak{L} \in L(\alpha)$, and $\mathfrak{\&}$ is also the annihilator of $f(v)$ in $A^{a, a}$. Since

$$
\mathbb{M}_{1} \cap A^{a, \alpha}=\mathfrak{L}=\mathbb{M}_{2} \cap A^{a, a},
$$

we have $\mathbb{M}_{1}=M_{2}=\phi^{-1}(\varrho)$, by Proposition 4.7. Thus $V_{1}$ and $V_{2}$ are equivalent $\Re$-modules, since each is equivalent to $B / \phi^{-1}(\varrho)$.

Finally, suppose that $W$ is an irreducible $A^{\alpha, \alpha} / B g^{\alpha}$-module, and let $w \in W$, $w \neq 0$. Regard $W$ as an $A^{a, a}$-module. Then the annihilator $\mathcal{L}$ of $w$ in $A^{a, a}$ is

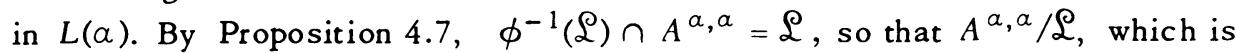
equivalent to $W$, is naturally embedded in the $B$-module $B / \phi^{-1}(\mathscr{Q})$. Since

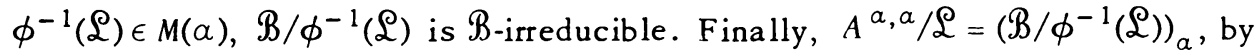
Proposition 4.4 applied to $V=\mathscr{B} / \phi^{-1}(\varrho), \beta=\alpha$ and $S=\left\{1+\phi^{-1}(\varrho)\right\}$. Q.E.D.

5. The absolutely irreducible case. Let the field $F$ be arbitrary. If $R$ is an algebra (over $F$ ) and $S$ is a subset of $R$, we denote by $R^{S}$ the centralizer of $S$ in $R$. Our aim in $\$ 5$ is to study the relationship between $B^{Q}$ and the algebras $A^{a, a}$, and to sharpen The orem 4.9. We begin with three general lemmas.

Lemma 5.1. Let $B$ be a full matrix algebra, and let $C$ be an arbitrary vector space (over F). Regard $B \otimes_{F} C$ as a left and a right $B$-module in the natural way. Then any subspace $D$ of $B \otimes C$ which is a left and right $B$-submodule is of the form $D=B \otimes(C \cap D)$. The conclusion bolds in particular if $C$ is an algebra and $D$ is a 2-sided ideal of the algebra $B \otimes C$.

Proof. $B$ may be regarded as a left and a right $B$-module under left and right multiplication, respectively, so that $B \otimes B$ acts as a space of operators on $B$. It is well known that this space of operators consists of all linear endomorph isms of $B$. In particular, $D$ is invariant under all operators $Q \otimes 1$, where $Q$ is any linear endomorphism of $B$.

Let $b_{1}, \cdots, b_{N}$ be a basis of $B$. Let $a=\sum_{i=1}^{N} b_{i} \otimes c_{i} \in D\left(c_{i} \in C\right)$. For all $i=1, \cdots, N$, let $Q_{i} \in \operatorname{End}_{F} B$ be such that $Q_{i} b_{i}=1$ and $Q_{i} b_{j}=0$ for $j \neq i$. Since $D$ is invariant under $Q_{i} \otimes 1$, we have $1 \otimes c_{i} \in D$. Q.E.D.

Lemma 5.2 (cf. [5, p. 118, Theorem 2]). Let $B$ be as in Lemma 5.1, and let $E$ be an arbitrary algebra containing $B$ as a subalgebra. Then $E=B \otimes_{F} E^{B}$ as algebras.

Proof. Let $E_{l}$ (resp., $E_{r}$ ) denote the left (resp., right) $B$-module induced by left (resp., right) multiplication of $B$ on $E$. Then $E_{l}$ is a direct sum of copies of the unique simple left $B$ module $V$, and $E_{r}$ is a direct sum of copies of the unique simple right $B$-module $V^{*}$. Regard $E_{l} \otimes E_{r}$ as a left and a right $B$-module in the obvious way. Then $E_{l} \otimes E_{r}$ is a direct sum of copies of $V \otimes V^{*}$. Let 


$$
X=\left\{x \in V \otimes V^{*} \mid b \cdot x=x \cdot b \text { for all } b \in B\right\} .
$$

Then it is well known that $X$ is one dimensional, and that $V \otimes V^{*}=B \cdot X$, since $V^{*}$ is isomorphic to the dual of $V$. Hence if we let

$$
Y=\left\{y \in E_{l} \otimes E_{r} \mid b \cdot y=y \cdot b \text { for all } b \in B\right\},
$$

then $E_{l} \otimes E_{r}=B \cdot Y$.

Now the multiplication map from $E \times E$ to $E$ induces a left and right $B$-module map $m: E_{l} \otimes E_{r} \rightarrow E$. Thus $m(Y) \subset E^{B}$, and so $E=B E^{B}$.

Let $\pi: B \otimes E^{B} \rightarrow E$ be the homomorphism induced by multiplication. Then $\pi$ is surjective by the above. But $\operatorname{Ker} \pi$ is a two-sided ideal of $B \otimes E^{B}$, and so

$$
\operatorname{Ker} \pi=B \otimes\left(\operatorname{Ker} \pi \cap E^{B}\right) \text {, }
$$

by Lemma 5.1. Since $\pi$ is injective on $E^{B}$, we must have $\operatorname{Ker} \pi=0$. Q.E.D.

Lemma 5.3. Let $B$ be as in Lemma 5.1, and let $C$ be an algebra. Let $X$ be a fixed irreducible $B$-module (so that $X$ is uniquely determined up to equivalence). Then the correspondence $Y \mapsto X \otimes_{F} Y$ ( $Y$ a C-module) induces a bijection from the set of equivalence classes of irreducible (resp., all) $C$-modules to the set of equivalence classes of irreducible (resp., all) $\left(B \otimes_{F} C\right)$-modules. The inverse of this bijection is given by $Z \mapsto \operatorname{Hom}_{B}(X, Z)(Z$ a $B \otimes C$-module).

Proof. Let $Z$ be a $B \otimes C$-module, and let $Y=\operatorname{Hom}_{B}(X, Z)$, regarded as a $C$-module. Then $X \otimes Y$ is a $B \otimes C$-module. Let $\phi: X \otimes Y \rightarrow Z$ be the linear map given by $x \otimes f \mapsto f(x)$. Then $\phi$ is a linear isomorphism. Indeed, write $Z$ as direct sum of copies of $X$, and choose a fixed $B$-isomorphism of $X$ onto each copy Since $X$ is absolutely irreducible under $B$, the resulting set $\left\{f_{j}\right\}$ is a basis of $Y$. Let $\left\{x_{i}\right\}$ be a basis of $X$. Then $\left\{x_{i} \otimes f_{j}\right\}$ is a basis of $X \otimes Y$, and $\phi\left(\left\{x_{i} \otimes f_{j}\right\}\right)$ is a basis of $Z$. Thus $\phi$ is a linear isomorphism. Since $\phi$ is clearly a $B \otimes C$ module map, $\phi$ is a $B \otimes C$-module isomorphism.

Conversely, let $Y$ be a $C$-module, and let $Z=X \otimes Y$, regarded as a $B \otimes C$ module. Then $\operatorname{Hom}_{B}(X, Z)$ is a $C$-module. Let $\psi: Y \rightarrow \operatorname{Hom}_{B}(X, Z)$ be the linear map given by $y \mapsto(x \mapsto x \otimes y)$. To show that $\psi$ is a linear isomorphism, choose a basis $\left\{y_{j}\right\}$ of $Y$. This determines a decomposition of $Z$ into a direct sum of copies of $X$, together with a fixed $B$-isomorphism of $X$ onto each copy; the $B$-isomorphism associated with $y_{j}$ is given by $x \mapsto x \otimes y_{j}$. Since $X$ is absolutely irreducible under $B$, this set of $B$-isomorphisms is a basis $\operatorname{Hom}_{B}(X, Z)$. Thus $\psi$ is a linear isomorphism, and hence a $C$-module isomorphism. This establishes the lemma, except for the irreducibility.

Let $Y$ be a $C$-module, and suppose that $Y_{1}$ is a proper nonzero $C$-submodule of $Y$. Then $X \otimes Y_{1}$ is a proper nonzero $B \otimes C$-submodule of $X \otimes Y$. Thus if 
$Y$ is not $C$-irreducible, then $X \otimes Y$ is not $B \otimes C$-irreducible. Conversely, let $Z$ be a $B \otimes C$-module, and let $Z_{1}$ be a proper nonzero $B \otimes C$-submodule of $Z$. Then $\operatorname{Hom}_{B}\left(X, Z_{1}\right)$ is a proper nonzero $C$-submodule of $\operatorname{Hom}_{B}(X, Z)$. Thus if $Z$ is not $B \otimes C$-irreducible, then $\operatorname{Hom}_{B}(X, Z)$ is not $C$-irreducible. Q.E.D.

We now make the assumptions of $\$ 2$, together with the assumption that the natural representation of $a$ on $b$ be finitely semisimple. In addition, we fix $a \epsilon$ $\hat{\mathfrak{Q}}$ and assume that $\alpha$ is an equivalence class of absolutely irreducible representations of $\mathbb{Q}$, so that $\mathbb{Q} / \mathbb{g}^{a}$ is a full matrix algebra.

Now $\mathfrak{Q} \subset A^{a, a}$ and $\mathfrak{B}^{\mathfrak{Q}}=\mathfrak{B}^{a} \subset A^{a, a}$, and we may identify the images of $\mathfrak{A}$

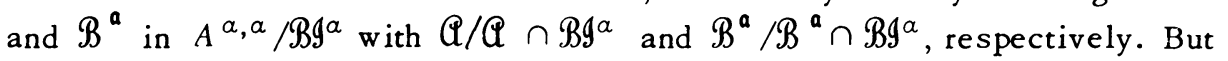
$\mathfrak{A} \cap \mathfrak{B g}^{a}=\mathfrak{g}^{a}$ by Lemma 2.2, so that $\mathfrak{A} / \mathfrak{A} \cap \mathfrak{B g}^{a}=\mathfrak{Q} / \mathscr{g}^{a}$.

Theorem 5.4. We bave

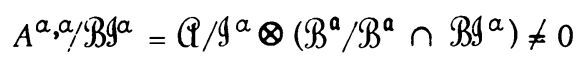

as algebras. In particular, $A^{a, a}=\mathfrak{C B}^{a}+B^{a}$.

Proof. By Lemma 5.2, we have

$$
A^{a, a} / \mathfrak{B g}^{a}=\mathscr{Q} / \mathscr{g}^{a} \otimes\left(A^{a, a} / \mathfrak{B g}^{a}\right)^{a} ;
$$

here the second factor denotes the centralizer of $a$ in the quotient $A^{a, a} / B^{a}$ of $a$-submodules of $\mathfrak{B}$ under the natural (adjoint) representation of $a$ on $\mathfrak{B}$. But since $b$ is finitely semisimple under $a$, the same is true of $B$, and hence of $A^{a, a}$. Thus

$$
\left(A^{a, a} / B^{a}\right)^{a} \simeq\left(A^{a, a}\right)^{a} /\left(A^{a, a}\right)^{a} \cap B^{a}=\mathcal{B}^{a} / \mathcal{B}^{a} \cap B^{a} \text {. Q.E.D. }
$$

Theore $\mathrm{m}$ 5.5. Let $V$ be a $\mathfrak{B}$-module, and fix an $\mathfrak{Q}$-module $X$ in the class $\alpha$. Then $\operatorname{Hom}_{\mathfrak{Q}}(X, V)=\operatorname{Hom}_{\mathfrak{Q}}\left(X, V_{a}\right)$ is a $\mathfrak{B}^{\mathfrak{a}} / \mathfrak{B}^{\mathfrak{a}} \cap \mathfrak{B}^{a}$-module by the action of $\mathfrak{B}^{\mathfrak{a}} / \mathcal{B}^{a} \cap \mathcal{B g}^{\alpha}$ on $V_{a}$, and the correspondence $V \mapsto \operatorname{Hom}_{\mathbb{Q}}(X, V)$ induces a bijection from the set of equivalence classes of all irreducible $B$-modules $V$ such that $V_{a} \neq 0$ with the set of equivalence classes of all irreducible $\mathfrak{B}^{a} / \mathfrak{B}^{a} \cap \mathfrak{B g}^{a}$-mod. ules. Moreover (cf. [4, p. 36, Corollary 2]), if $V$ is sucb a ß-module, then $V_{a}$ is invariant and irreducible under $\mathfrak{A B}^{a}$ (a subalgebra of $\mathfrak{B}$ independent of $\alpha$ ), and the equivalence class of $V$ is determined unquely by the equivalence class of the $\mathfrak{A B}{ }^{a}$-module $V_{a}$.

Proof. The bijection assertion follows immediately from Theorems 4.9 and 5.4, together with Lemma 5.3. The irreducibility of $V_{a}$ under $\mathfrak{A B}^{a}$ is an immediate consequence of Proposition 4.5 and the second assertion of Theorem 5.4, and the last assertion follows from Theorem 4.9 and the second assertion of Theorem 5.4. Q.E.D. 


\section{BIBLIOGRAPHY}

1. N. Bourbaki, Éléments de mathématique. XXVI. Groupes et algèbres de Lie. Chap. 1: Algèbres de Lie, Actualités Sci. Indust., no. 1285, Hermann, Paris, 1960. MR 24 \#A2641.

2. R. Godement, A theory of spherical functions. I, Trans. Amer. Math. Soc. 73 (1952), 496-556. MR 14, 620.

3. Harish-Chandra, Representations of a semisimple Lie group on a Banach space. I, Trans. Amer. Math. Soc. 75 (1953), 185-243. MR 15, 100.

4. - Representations of semisimple Lie groups. II, Trans. Amer. Math. Soc. 76 (1954), 26-65. MR 15, 398.

5. N. Jacobson, Structure of rings, Amer. Math. Soc. Colloq. Publ., vol. 37, Amer. Math. Soc., Providence, R.I., 1956. MR 18, 373.

6. B. Kostant, On the existence and irreducibility of certain series of representations, Bull. Amer. Math. Soc. 75 (1969), 627-642. MR 39 \#7031.

7. J. Lepowsky, Algebraic results on representations of semisimple Lie groups, Trans. Amer. Math. Soc. 176 (1973), 1-44.

DEPARTMENT OF MATHEMATICS, BRANDEIS UNIVERSITY, WALTHAM, MASSACHUSETTS 02154

DEPARTMENT OF MATHEMATICS, MICHIGAN STATE UNIVERSITY, EAST LANSING, MICHIGAN 48823

Current address (J. Lepowsky): Department of Mathematics, Yale University, New Haven, Connecticut 06520

Current address (G. W. McCollum): Department of Mathematics, Tufts University, Medford, Massachusetts 02155 